\title{
Cloud Computing: A New Paradigm in IT that has the Power to Transform Emerging Markets
}

\author{
San Murugesan
}

\begin{abstract}
Cloud computing offers utility-like availability of computing resources and applications via the Internet at low cost. It has the power to transform enterprises in emerging markets offering access to advanced IT infrastructure and applications that many of them couldn't afford. An informed understanding of cloud computing and its benefits, limitations and risks are the keys to successfully embrace the opportunities this new computing paradigm offers. This article presents an overview of cloud computing concepts, cloud services, cloud hosting models, and applications. It also identifies potential risks and discusses the prospects of clouds and what businesses and individuals can do to successfully embrace cloud computing.
\end{abstract}

Index terms-Cloud Computing, Virtualisation, Software as a Service, Platform as a Service, Infrastructure as a Service

\section{INTRODUCTION}

$\mathrm{C}$ omputing landscape - both enterprise and personal - is changing again with the emergence of cloud computing. In its evolution over the past four decades, computing has passed through several stages -- from mainframe computers to minicomputers to personal computers to network computing, client-server computing, and distributed computing. Now, coming full circle, computing is migrating outward to the clouds, to distant computing resources reached through the Internet. In the cloud computing model, applications and computing resources are provided to users as a service through the Internet (i.e., "the cloud"), similar to utilities like electricity supplies. Users can use computing resources when and where they need them and in the amount they need, and pay for the resources used. Cloud computing offers huge computing power, on-demand scalability, and utility-like

This is an abstract version of the keynote presented at ICTer2010 Conference, Colombo.

San Murugesan is Adjunct Professor at the University of Western Sydney in Australia and Director of BRITE Professional Services.

He served in academia and industry for a long period and has published widely. His current areas of interest are green IT, cloud computing and IT in emerging markets. He is co-editor of the upcoming book, Harnessing Green IT: Principles and Practices (Wiley, 2011) and editor of Handbook of Research on Web 2.0, 3.0, and X.0: Technologies, Business, and Social Applications (Information Science Reference, 2009). Dr Murugesan serves as Associate Editor-in-Chief of IEEE IT Professional magazine, and green technology and IT in emerging markets editor for the magazine. He also serves on the editorial board of several other international Journals, IEEE Technical Steering Committee on Green Computing and the advisory board of Global Science and Technology Forum. He is a Fellow of Australian Computer Society and Senior of IEEE Computer Society.

He can be contacted: san1@internode.net availability at low cost and is a radically new IT delivery and business model.

Cloud computing causes paradigm shift in how we deliver and use computing resources and applications and it is poised to have profound impact on businesses, commerce and individuals. It can reduce significantly information technology costs - by as much as 50 to 80 percent - and offer businesses better agility and flexibility. As a result cloud computing is gaining keen interest among IT vendors, professionals and users - business, governments and individuals. Cloud service delivery model is particularly attractive to business and individual users in emerging markets. The rise of the cloud is more than just another IT platform shift; clouds will transform not only the IT industry but also the emerging markets, and the transformation will be profound. Clouds presents many opportunities for emerging markets to profoundly improve the way people communicate, share information and work and companies operate. Emerging markets can embrace clouds offering new kinds of applications. Even a small business can suddenly serve millions of customers all around the world and the flexibility and versatility clouds enables companies to enter new markets quickly with very little overhead costs.

In this paper, we examine cloud computing concepts, cloud services, cloud hosting models, and applications. We also identify potential risks and discuss the prospects of clouds and what businesses and individuals can do to successfully embrace cloud computing. Finally, it also discusses clouds' prospects and implications to businesses and individuals.

\section{COMPUTING}

The "Cloud" is an evolution of distributed computing and of the widespread adaption of virtualization and service oriented architecture (SOA). In cloud computing, IT-related capabilities and resources are provided as service, via the Internet and on-demand, accessible without requiring detailed knowledge of the underlying technology. Depending on one's perspective, clouds can be described in different ways [see Geelan 2009] and hence the term cloud computing remains fuzzy and carries several definitions.

- "Cloud computing is a model for enabling convenient, on-demand network access to a shared pool of configurable computing resources (e.g., networks, servers, storage, applications, and services) that can be rapidly provisioned and released with minimal management effort or service provider interaction" [1]. 
- "A style of Computing where scalable and elastic IT capabilities are provided as a service to multiple customers using Internet technologies" - Gartner.

Key benefits of computing clouds are reduced capital and operational costs, improved flexibility and agility, ubiquitous access, and easier and quicker application development and deployment [2].

\section{A. Cloud Structure}

A computing cloud is a massive network of computing resources interconnected in a grid running in parallel, harnessing the resources of each to provide huge computing and storage capacity. The cloud is accessible from a PC or a smart mobile phone, over the Internet through a Web browser. Users' request is processed by the cloud's system management software which manages the cloud resources and assigns and monitors users' processing tasks. The system software finds suitable resources to perform the requested task and calls the system's provisioning service which request and block necessary resources in the cloud and launch the appropriate application(s). Once an application is launched, the cloud's monitoring and metering functions track resource usage and bills the users for the services used. Automatic dynamic management of the cloud's tasks and monitoring and reporting the usage are key aspects of cloud computing.

\section{B. Attributes of Computing Clouds}

Computing clouds have several distinguishing attributes. They:

- have massive resources at its disposal and can support several users simultaneously.

- $\quad$ support on-demand scalability of users' computational needs.

- offer ubiquitous access. Stored data and applications are accessible by authorized users anywhere, anytime.

- facilitate data sharing, enterprise-wide data analysis, and collaboration.

- can self-reconfigure providing continuous availability in case of failure of its computing resources.

- offer enhanced user experience via a simplified Web browser user interface.

\section{What's New?}

While the clouds draw on some of the older foundations of IT such as centralized shared resources pooling, concept of utility computing and virtualization, they incorporate new mechanisms for resource provisioning and dynamic scaling. Further, it adopts new business and revenue models and incorporates monitoring provisions for charging for the resources used. Cloud computing became viable for wider offering and adoption only recently with the adoption of broadband Internet access and advances in virtualization and data centre design and operation as well as the crucial philosophical and attitude change by both the IT vendors and users.

\section{A Paradigm Shift}

Cloud computing represents a paradigm shift - transition from computing-as-a-product towards computing-as-a-service. It's a transition from buying hardware and software as products which we install, configure, use and maintain to using applications and computing infrastructure in the clouds as a service, paying on the go for the resources used. With cloud computing, you aren't tied to a particular computer or a specific private network to run or access your applications and data. Further, cloud computing makes it easier for group members in different locations to collaborate both synchronously and asynchronously. It also facilitates a variety of mobile computing applications which are poised for major uptake.

\section{Cloud COMPuting SERVICE Models}

Cloud services can be classified into four broad categories: software as a service (SaaS), platform as a service (PaaS), infrastructure as a service (IaaS), and other Internet-based Services (IBS). Each service category can be used independently or used in combination with others.

\section{A. Software as a Service}

In the SaaS model, end-user applications are hosted by a cloud vendor and delivered as a service to customers across the Internet on some form of "on-demand" billing system or free as in some personal applications. This model eliminates the need to locally install and run the application on the user's computer, and thereby also alleviates the users from the burden of hardware and software maintenance and upgrades. The software license is not owned by the user. Costs to use the service become a continuous expense rather than an up-front huge capital expense at the time of purchase. Examples of SaaS include Webmail, Google Apps, Force.com CRM, Quicken online accounting, NetSuite's Business Software Suite, Sun Java Communications Suite and Paychex payroll management system. SaaS clouds are also called software clouds.

\section{B. Platform as a Service}

In the PaaS model, application development platforms and middleware systems are hosted by a vendor and offered to application developers, allowing them to simply code and deploy without directly interacting with the underlying infrastructure. The platform provides most of the tools and facilities required for building and delivering applications and services - workflow facilities for application design, development, testing, deployment, and hosting, as well as application services such as Web service integration, database integration, security, storage, application versioning, and team communication and collaboration. Examples of PaaS include Google App Engine, Microsoft Azure, Amazon's Web 
Services and Sun Microsystems NetBeans IDE. The PaaS clouds are also called platform clouds and cloudware.

\section{Infrastructure as a Service}

In an IaaS cloud, raw computer infrastructure, such as servers, CPU, storage, network equipment and data centre facilities are delivered as a service on demand. Rather than purchasing these resources, clients get them as a fully outsourced service for the duration they need them. The service is billed according to the resources consumed. Amazon Elastic Compute Cloud (EC2), GoGrid, and FlexiScale are some of the examples of IaaS clouds. These types of clouds are also called utility computing or infrastructure clouds.

\section{Other Internet-Based Services}

Storage, middleware, collaboration, and database capabilities are other Internet-based services that clouds offer directly to users. With cloud storage, data is stored in multiple third-party servers, rather than on dedicated servers used in traditional networked storage, and users see a virtual storage. The actual storage location may change as the cloud dynamically manages available storage space; however, the users see a static location for their data.

Data storage is a common infrastructure use of cloud computing. Key advantages of cloud storage are cost, data safety and availability. Data stored in a cloud is safe against accidental erasure or hard-drive failures as a cloud keeps multiple copies of data across multiple physical machines continually. If one machine crashes, the data that was in that machine can be retrieved from other machine(s) in the cloud. Cloud vendors generally offer more powerful security measures than what a small business could afford. Enterprise data storage in clouds, however, raises some concerns which are discussed later.

\section{Cloud Computing Hosting Models}

Based on where the clouds are deployed and by whom, computing clouds are classified into five categories: public clouds, private or internal clouds, virtual private clouds, vertical or community clouds, and hybrid clouds.

\section{A. Public Clouds}

A most common and widely known form of clouds, public clouds are open to anyone and the cloud infrastructure and applications are owned by the cloud provider - the organization that offers the cloud services. Public cloud services are usually offered on a pay-per-usage model; some applications on public clouds can be used free of charge.

\section{B. Private or Internal Clouds}

These are clouds provided and controlled by an enterprise behind its firewall. Unwilling to head into public clouds because of concerns surrounding them and compliance requirements, some enterprises have begun to deploy their own cloud computing environment for their exclusive use to gain operational efficiencies and to effectively use their existing resources.

\section{Virtual Private or External Private Clouds}

These are segments of a public cloud designated for a user with added provisions for meeting specific security and compliance requirements. They provide users more control over the resources they use than in a pure public cloud. An example of this type of cloud is Amazon's Virtual Private Cloud.

\section{Vertical Cloud or Community Clouds}

These clouds are optimized for use by a particular industry sector or a group of users meeting their specific requirements to address issues that are crucial to them. AcademyOne's Academic Navigator aimed at academics and students and Asite Solution's applications specifically designed for the construction industry are examples of these types of clouds.

\section{E. Hybrid Clouds}

A hybrid cloud is a combination of two or more of the above cloud hosting models. In this model, an enterprise makes use of both public and internal clouds deploying its less critical, low-risk services on public clouds and businesscritical core applications on its internal cloud. A hybrid model allows for selective implementation addressing security, compliance and loss of control concerns as well as enabling adoption of public clouds that offer cost benefits and more application options.

\section{F. Choosing Your Cloud}

A major decision that IT managers and enterprises have to make is the cloud type - public clouds, internal clouds, or variations of them - that is well suited for their applications. To arrive at a better decision, they have to understand the differences between these deployments, and understand the risks associated with each in the context of characteristics and requirements of their applications. Further, they also have to consider [3]:

- performance requirements, security requirements, and cloud service availability and continuity;

- amount of data transfer between the user and the clouds and/or between the clouds;

- $\quad$ sensitive nature of the applications;

- control of their application and data;

- total cost involved;

- trust on the external cloud providers;

- terms and conditions imposed by the external cloud providers; and

- in-house technical capabilities.

\section{PRos AND CONS OF Cloud COMPUTING}

Cloud computing offers several benefits to users - both individual and enterprises. But it also has limitations and poses some risks the impact of which depends on the 
application type and liabilities involved. In embracing cloud computing, therefore, users must understand, acknowledge, and address these limitations and risks.

\section{A. Benefits of Cloud Computing}

Clouds offer the following benefits:

- Lower operational/service cost to users - they pay for what they use

- On-demand scalability to meet peak and uncertain computing demands

- Access to applications from anywhere, anytime, any device

- Shared access to data/application supporting collaboration and teamwork

- $\quad$ Ease of and quicker application deployment

- Freedom from being tied up to a single computer

- Increased data safety than most businesses can afford and manage in their own on-premise IT system

Public clouds eliminate significant capital expenses for hardware and up-front license fees for software as well as the headaches of hardware and software maintenance and upgrade by the users. Cloud applications can be deployed instantly and simultaneously to thousands of users in different locations around the world, and can also be regularly updated easily. Further, as clouds provide improved business continuity and data safety, they are particularly attractive to small and medium-size enterprises as well as enterprises in natural disaster prone areas. Application developers can use computing clouds to try their ideas without having to invest on their own infrastructure.

\section{B. Limitations of Cloud Computing}

The general limitations of cloud computing are:

- the need for a constant, high-speed network access to connect to clouds;

- the possibility of slow response at times due to increased traffic on the network or higher load on the computers in the cloud;

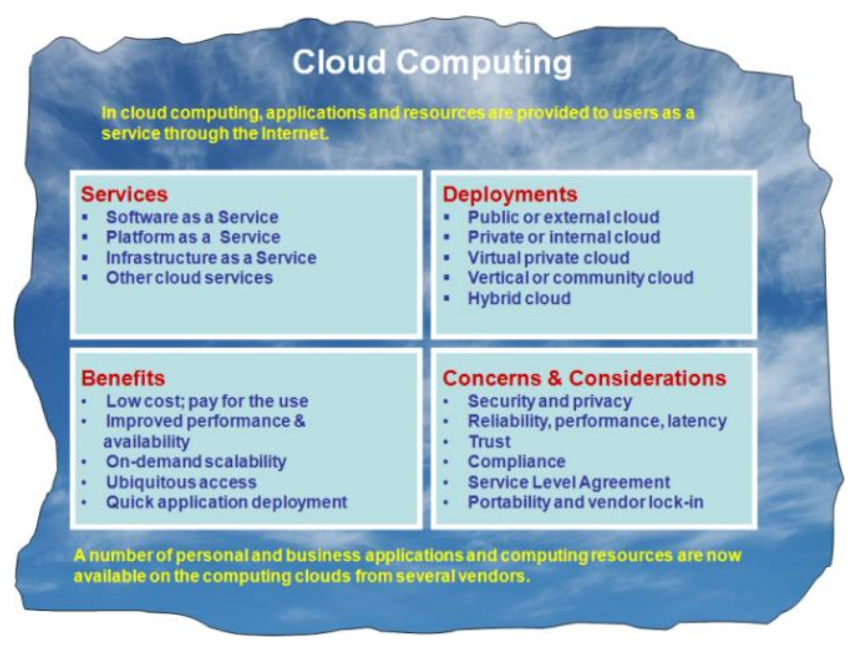

Fig. 1. A snapshot of cloud computing
- the security of the data on external clouds;

- risks of unauthorized access to users' data; the loss of data due to cloud failure (despite replication across multiple machines); and

- concerns regarding reliability and continued availability of services by cloud providers.

For a snapshot of cloud computing, refer to Figure 1. Table 1 highlights key technologies that support cloud computing and features and benefits of cloud computing.

TABLE I

Cloud COMPUTING PARADIGM

\begin{tabular}{|c|c|}
\hline Technology & $\begin{array}{ll}\text { - } & \text { Virtualization } \\
\text { - } & \text { Provisioning } \\
\text { - } & \text { Service orientation } \\
\text { - } & \text { Metering services } \\
\text { - } & \text { Load balancing } \\
\text { - } & \text { Web services API } \\
\text { - } & \text { Redundancy and fail over } \\
\text { - } & \text { Security }\end{array}$ \\
\hline Features & 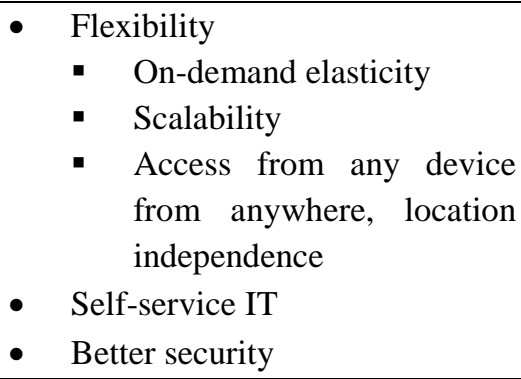 \\
\hline $\begin{array}{l}\text { Business } \\
\text { Benefits }\end{array}$ & $\begin{array}{l}\text { - } \text { On-demand provisioning } \\
\text { - } \quad \text { Ability to easily meet evolving } \\
\text { IT requirements } \\
\text { - } \quad \text { Immediacy - quick to deploy } \\
\text { applications } \\
\text { - } \quad \text { Effective utilization of } \\
\text { resources } \\
\text { - } \quad \text { Service level contract (SLA) }\end{array}$ \\
\hline $\begin{array}{l}\text { Economic } \\
\text { Benefits }\end{array}$ & $\begin{array}{ll}\text { - } & \text { Utility-based, pay-as-you go } \\
\text { model } & \\
\text { - } & \text { No long term } \\
\text { contract/commitments } & \\
\text { - } & \text { No capital expenses } \\
\end{array}$ \\
\hline
\end{tabular}

\section{Cloud APPliCATIONS}

The most popular cloud-based applications are Web mail and document management and sharing systems such as Google Docs, calendars, and contact management, Microsoft Office Live Workspace, Apple MobileMe, and Adobe Photoshop Express. Businesses cloud applications include customer relationship management (CRM) tools, payroll processing, human relationship management and VoIP in clouds. Enterprise cloud applications and services are provided by a range of vendors including Salesforce.com, NetSuites and Fonality, EMC, HP, IBM, Oracle, SAP, 
Accenture, and Infosys. Clouds help smaller companies access applications and services that they couldn't have access to otherwise but were available only to larger enterprises. One of the cloud-based services used by most internet users is online storage and backup services provided by vendors such as Carbonite, iDrive, Rackspace, and Google. Several businesses of all sizes in different sectors have used clouds for different kinds of applications and derived cost and other benefits.

For example, City of Orlando cut costs by over 66 percent by moving email and application into the cloud [4]. At BP, cloud computing solved a short-term capacity problem that its existing data centre had no cost-effective way of addressing [5]. Lilly used Amazon's EC2 service cloud to deliver speed and flexibility to its researchers for doing data analysis, rather than investing on 50 servers needed to do the analysis the researchers were interested [5]. The New York Times harnessed 100 virtual cloud servers to convert 11 million documents to PDF and archived the documents on the cloud, spending only $\$ 240$ [6].

Future Group, India's largest retailer, is using cloud computing to support data warehousing and analytics for its multi-format chain of retail stores. The retailer uses clouds to manage its customer loyalty program which involves storage and analysis of millions gigabytes of data [7].

Major companies now use the cloud to provide affordable specialised IT services to small and medium enterprises. For instance, GE Healthcare has launched a software service that allows physicians to manage the medical side of their practice. Physicians can obtain and share data with other medical practitioners, access decision support tools and prepare and share medical reports about their patients. The service also lets their patients to schedule appointments and request prescriptions and access their laboratory results [8, 10].

Most businesses now use some form of cloud computing. According to Mimecast Cloud Adoption Survey [9], 51 percent of enterprises have already adopted some form of cloud computing. 66 percent of enterprises are now thinking of adopting cloud computing. Companies that used cloud said they were very satisfied with their cloud experiences: 74 percent of users claimed that they were using resources better; 73 percent reported a reduction in infrastructure costs, and 72 percent said that end-user experience had improved.

An Accenture report [10] highlights some of the others business applications of cloud. Government agencies in the US, UK and other countries are leaning toward cloud computing and software-as-a-service solutions as they try to meet the dual goals of containing costs and modernizing technology. The Whitehouse Office of Management and Budget has announced that, from now on, cloud computing would be the "default approach to IT" for US government agencies. The cloud-first policy [11] is expected to reduce over 2000 data centre infrastructure by as much as 40 percent, lowering costs, improving security and performance, and speeding up the deployment of new applications.

\section{Cloud CONCERNS AND REMEDIATION}

Despite its promises, cloud computing's mainstream adoption is constrained by perceived and real barriers and concerns. Security and privacy are two of top concerns in moving into clouds followed by reliability and availability of cloud services, as well as adherence to compliance requirements, where applicable. External clouds raise additional concerns about loss of control and sharing data outside the enterprise firewall, and on potential hike in cost of services by cloud vendors.

Many people think that because they don't know where their data is stored remotely and since the applications are accessed over the Internet, cloud applications are insecure, and believe that if the data and application are physically housed in computers under their control they can protect them better. But this is not necessarily the case as economies of scale allow cloud providers to offer for more sophisticated security, disaster recovery, and service reliability than an individual institution, particularly small enterprise, can deploy on its own. However, recent security breaches at Salesforce.com, epic.org and Google Docs, to name a few, attest the ongoing concerns on cloud security and serve as reminders to be cautious.

Cloud computing security concerns and requirements can differ considerably among the stakeholders - end-user service consumer, cloud service provider and cloud infrastructure provider and are determined by the specific services they provide or consume. The Cloud Security Alliance has identified seven top cloud security threats and outlined impact of those threats as well as remediation for them [12].

Many enterprise computing applications must meet compliance requirements which depend on the type of business and customer base. Although not a guarantee, to better ensure desired level of service delivery and to limit the liabilities, service level agreements (SLAs) with the cloud vendor is highly recommended when consuming cloud services. A cloud SLA specifies terms and conditions as well as expectations and obligations of the cloud service provider and the user. By careful planning and user's requirements built into service providers' cloud offerings, both the cloud vendors and users can reduce risk and reap the rewards of cloud-based hosted services.

\section{MigRATING TO CLOUDS}

To embrace cloud computing a new mindset is needed. To successfully use and benefit from clouds, an enterprise must prepare itself strategically, culturally and organizationally, and take a holistic view of cloud computing. It must develop its strategic plan and follow a phased, pragmatic step-by-step approach that provides a business context for its cloud adoption. It must choose a cloud option that is appropriate for the application in consideration, as outlined earlier, and manage the risks of migrating to clouds by applying safeguards and provisions available [13]. Moving into clouds 
is not just about technology, the cloud migration should also factor in the role of people, processes and services, and the change management process. Migration to clouds will also demand a new kind of IT management and governance framework.

\section{Cloud PROSPECTS AND IMPLICATIONS}

Computing clouds is a powerful change-agent and an enabler. Soon the core competency for most enterprises would be on using IT services and infrastructure that cloud computing offers as hosted services, not building their own IT infrastructure. Cloud computing is already transforming the way we think about computing environments and can drastically improve access to information as well cut IT costs.

Ongoing developments -- increasing maturity of clouds, introduction of new cloud computing platforms and applications, growth in adoption of cloud computing services, and the emergence of open standards for cloud computing will boost cloud computing's appeal to both cloud providers and users. More personal productivity applications will appear as software services.

Clouds will enable open-source and freelance developers to deploy their applications in the clouds and profit from their developments. As a result, more open source software will be published in clouds. The SaaS model of cloud computing has reached the tipping point and will be widely adopted as an online service. PaaS and IaaS models have matured for mainstream adoption.

Cloud Computing will profoundly change the way people and enterprises use computers and their work practices as well as how companies and governments deploy their computer applications. Clouds will also help close the digital divide prevalent in emerging and underdeveloped economies. They also help save our planet by presenting a greener computing environment.

Driven by economic imperatives and the promise of flexibility and convenience, cloud computing will gain wider acceptance and adoption. Barriers to enterprise adoption of cloud computing will gradually be lowered or removed as IT professionals and the IT industry addresses these issues. Government regulations and compliance requirements will also be amended to embrace cloud computing.

Like the Internet, cloud computing is a transformational technology. It'll mature rapidly as vendors and enterprises come to grip with the opportunities and challenges that it presents. It's time to explore, experiment and embrace cloud computing as relevant.

\section{A. Cloud Implications}

The hosted computing model creates opportunities for all IT and non-IT enterprises, researchers and individuals. It creates new possibilities for businesses. There will be new investments that create new business models, new opportunities to start and form businesses. Researchers would be better able to run experiments quickly on clouds, share their data globally, and perform complex analysis and simulations. A whole new set of courses focused on cloud computing would be offered by universities and training institutions.

However, a pertinent question that arises among many IT professionals is: Will cloud computing kill IT jobs? It seems many IT professionals particularly those work on on-premise IT systems are afraid of losing their jobs because of cloud computing. While some might lose their current job, they might get absorbed in other roles. So they should be looking to evolve their roles. They will need to learn about clouds, how to deploy and manage applications on the clouds and minimize risks, and how to work with cloud providers and other functional units within their enterprise. They may also be required to help others in their enterprise understand the opportunities offered by the clouds and the limitations and risks of new model. They may need to master their people skills as cloud computing inevitably brings together different stakeholders and their interests. Also there will be need for people who understand the technical advances in clouds and the cloud-based offerings to spread the cloud around the globe, for developing new kinds of applications, and for creating innovations in IT.

\section{Demystifying The MythS OF Cloud COMPUTING}

There are few myths that have risen due to confusion regarding the cloud. In the following we examine and demystify them [14].

\section{- Myth \#1 - Cloud isn't secure}

Ensuring security of their cloud services is always the top priority and competitive necessity for cloud providers. Cloud providers uses a range of security measures and strategies, including physical data centre security, separation of the network, isolation of the server hardware, and isolation of storage that enterprise data centres have used for long, and a lot more including $24 \times 7$ monitoring. For instance, cloud providers such as Amazon.com maintains packet-level isolation of network traffic and support industry-standard encryption. Most companies and individuals users don't have the luxury of dedicating enough resources on security like cloud providers. In view of the scale, cloud computing provider do invest in security controls and countermeasures and provide better security than almost any small and medium size company could afford.

- Myth \#2 - Cost is the only cloud advantage

The reality is cost is just one of them, the more important advantage is the ability to move more quickly and accelerate time-to-market. For a developer in an organization to get a server to do an experiment or just expand a project, might take a few months. With a cloud he can get access to large amounts of server capacity in minutes to expedite development work and at very low cost. 
- Myth \#3 - You should move all infrastructure to the cloud in one step

For a start-up it is advisable to move all infrastructure to cloud in one go. For enterprises that have new development it is easy to build it on top of the cloud and quickly take advantage of those benefits. Most major enterprises move more methodically by picking a diverse set of initial applications to try as proofs of concepts in the cloud. They run them for a few months to see how the cloud is different and understand how to operate in the cloud before moving more of their applications. Then follow it with a cloud migration plan.

- Myth \#4 - One can have all the benefits of the cloud with only a private cloud

The reality is private or internal clouds incur high capital and ongoing high maintenance costs. Special expertise is needed to implement and maintain a private cloud. Cloud computing allows companies to focus their capital and resources on innovations to accelerate their time to market, rather than running and maintaining the undifferentiating heavy lifting of infrastructure.

\section{Clouds FOR EMERGING MARKETS}

The ability to access software and computing power through public clouds means that many firms will not need to build proprietary systems or purchase expensive hardware. Cloud computing could give emerging market companies an opportunity to leapfrog over their rivals in developed countries. According some estimates IT professionals spend $70 \%$ of their time maintaining systems and only $30 \%$ of their time creating strategic value. Cloud computing frees the IT department from legacy issues and allows it to focus on innovative ideas to create competitive advantage.

Executives from businesses in developed countries appear more cautious about the opportunities that cloud computing bring to the businesses than their entrepreneurial counterparts in emerging regions. According to a survey, $70 \%$ of firms in the developing world are re-appraising their cloud platforms, compared with only $46 \%$ of firms in the developed world.

\section{A. Opportunities to Emerging Markets}

Clouds provide businesses and individuals access to advanced IT infrastructure and applications that many of them couldn't afford otherwise. They can offer productivity gains and better business continuity in the event of failures and natural disasters, besides cutting costs. They can also facilitate creation of 'Connected Enterprises' and fosters innovation supporting creating and deploying new applications on established cloud platforms quickly and cost effectively. There are now a growing number of cloud applications for virtually every aspect of a business' and individual's activities.

Cloud computing promises to speed application deployment, increase innovation, and lower costs. It also can transform the way we design, build, and deliver applications, and increase business agility. One can embrace clouds in a variety of ways to gain significant benefits and to offer applications that were not feasible otherwise. The developing world must exploit the opportunities offered by cloud computing while minimizing the associated risks. Thereby they can have access to advanced IT infrastructure, data centres, and applications, and also leverage the power clouds/IT for their benefit. In fact, developing economies could catch up with developed countries as the cloud gives them access to the same IT infrastructure, data centres, and applications.

Cloud computing could be applied in a range of areas: eCommerce, E-Business, E-Supply Chain Management; EEducation, E-Health, E-Governance, telework/ telecommuting, Collaboration, Community building and emergency response. Although the potential of cloud computing is vast and compelling, few in the industry can fully grasp its true impact on society. Adoption of computing clouds calls for new mindset: move from $\mathrm{P}$ to $\mathrm{S}$ - product to services

\section{B. Supporting Trends}

Several initiatives and developments support the emergence and adoption of clouds in emerging markets. Multinational companies are triggering the cloud industry's evolution in the developing world by establishing cloud/data centres and promoting clouds. Efforts by governments and international organizations to narrow digital divide and data divide can now better exploit the clouds. Growing middle class and IT-savvy new generations are keen on using IT and don't hesitate to compute in the clouds, like the previous generations.

Mobile Internet and Clouds form a powerful link. Twothirds of the globe's mobile phone users ( 4.6 billion) live in emerging markets, and with the growth of smart phones with wireless broadband, mobile Internet and cloud based applications will become more pervasive.

\section{CONCLUSION}

Cloud computing is positioning itself as a new platform for delivering information infrastructures and a range of computer applications for businesses, governments, charities and individuals as IT services. Cloud customers can then provision and deploy these services in a pay-as-you-go fashion and in a convenient way while saving huge capital investment in their own IT infrastructures. Clouds are evoking a high degree of interest - both in developed and emerging markets - though challenges such as security and privacy remains to be fully addressed.

The rise of the cloud is more than just another platform shift. It will transform the IT industry, but it will also profoundly change the way people work and companies operate. It will allow digital technologies to penetrate everywhere and leverage the economy and society particularly in emerging markets. 


\section{REFERENCES}

[1] "NIST Definition of Cloud Computing," January 2011. http://csrc.nist.gov/publications/drafts/800-145/Draft-SP-800-

145_cloud-definition.pdf.

[2] S. Murugesan, "Cloud Computing: A New Paradigm in IT," Cutter Business Intelligence Executive Report, Vol. 9, No. 2, 2009.

[3] B. Claybrook, "Cloud vs. in-house: Where to run that app?," Computer World, 11 March 2010 http://www.computerworld.com/s/article/9162542/Cloud_vs._in_house_ Where_to_run_that_app_?

[4] C. Cross, "City of Orlando: cutting costs while advancing our infrastructure in just two months," March 25, 2010. http://googleenterprise.blogspot.com/2010/03/city-of-orlando-cuttingcosts-while.html.

[5] D.Neal, et al., "Cloud rEvolution: A Workbook for Cloud Computing in the Enterprise,", Vol. 4, CSC Leading Edge Forum, 2010. http://assets1.csc.com/lef/downloads/LEF_2010CloudRev_Vol4_Workb ook.pdf.

[6] Introduction to Cloud Computing architecture, Sun Microsystems, June 2009.

http://eresearch.wiki.otago.ac.nz/images/7/75/Cloudcomputing.pdf.

[7] "Future Group Sets the Standard for Retail Analytics with Greenplum Database," $\quad$ PRWeb, 8 June 2009 www.prweb.com/pdfdownload/2505804.pdf.

[8] B.T. Horowitz, "GE SAAS Offering Eases Electronic Medical Records Management," www.eweek.com, June 15, 2010.

[9] "Loudhouse Research: Cloud Barometer Survey 2010." www.mimecast.com/barometerresearch2010.

[10] J.G. Harris and A.E. Alter, Cloudrise: Rewards and Risks at the Dawn of Cloud Computing, Accenture, Nov 2010. www.accenture.com/SiteCollectionDocuments/PDF/Accenture_Cloudri se_Rewards_and_Risks_at_the_Dawn_of_Cloud_Computing.pdf.

[11] V. Kundra, Federal Cloud Computing Strategy, Feb 2011 www.cio.gov/documents/Federal-Cloud-Computing-Strategy.pdf.

[12] Top Threats to Cloud Computing V1.0, Cloud Security Alliance, 2010. http://cloudsecurityalliance.org/topthreats/csathreats.v1.0.pdf

[13] I. Gotts, "Assessing the Risks of Migrating Your Offering to the Cloud," Cloud Computing Journal, 2010 http://www.cloudbook.net/resources/stories/assessing-the-risks-ofmigrating-your-offering-to-the-cloud.

[14] "Demystifying the Myths of Cloud Computing," 2010 www.datacenterdynamics.com/focus/archive/2011/08/demystifying-themyths-of-cloud-computing. 